\title{
Análise retrospectiva: o que denota os exames citopatológicos da unidade CEPS
}

O câncer do colo útero (CCU) é uma das causas de morte mais frequente em mulheres em todo o mundo, o exame de triagem é indicado para mulheres de faixa etária de 25 a 64 anos, que já iniciaram atividade sexual. Um fator que predispõe ao desenvolvimento dessa patologia é a infecção pelo papilomavírus humano (HPV). A realização do exame preventivo é uma estratégia eficaz na detecção precoce de lesões que precedem o CCU. Tem como objetivo em apresentar a prevalência de alterações dos resultados de exames citopatológicos das pacientes que realizaram consulta de enfermagem na clínica. Estudo de pesquisa de campo, com metodologia quantitativa e abordagem descritiva. Amostragem foi feito em 245 laudos de exames preventivos realizados no ano de 2018 a 2020 na unidade CEPS em Guaraí-TO. As atípicas celulares estavam presentes em 2,45\%, sendo (97,55\%) negativo para lesão intraepitelial. Com relação à idade, $(30,61 \%)$ das mulheres foi menor de 30 anos. A adequabilidade do material coletado apresentou amostra satisfatória em $100 \%$ dos registros. Quanto às alterações celulares benignas, a inflamação esteve presente em (70\%) 21. Sobre a escolaridade das mulheres, ensino médio completo predominou com (30,61\%). 0 agente microbiológico predominante foi Lactobacillus sp. com $(80,82 \%)$ dos resultados. As lesões intraepiteliais apresentaram baixa prevalência, em comparado com algumas literaturas. Percebe-se, que a saúde das pacientes da unidade CEPS de Guaraí, exibe boa adequabilidade, visto que os números de agentes que causa infecção estão reduzidos.

Palavras-chave: Exame Citopatológico; Mulher; Colo do Útero.

\section{Retrospective analysis: what does the cytopathological examinations of the CEPS unit}

\begin{abstract}
Cervical cancer (CC) is one of the most frequent causes of death in women worldwide; the screening test is indicated for women aged 25 to 64 years, who have already started sexual activity. One factor that predisposes to the development of this pathology is infection by human papillomavirus (HPV). The performance of the preventive exam is an effective strategy in the early detection of lesions that precede the CC. It aims to present the prevalence of changes in the results of cytopathological exams of patients who underwent nursing consultation at the clinic. Field research study, with quantitative methodology and descriptive approach. Sampling was carried out in 245 reports of preventive exams carried out in the year 2018 to 2020 at the CEPS unit in Guaraí-TO. The atypical cells were present in $2.45 \%$, being $(97.55 \%)$ negative for intraepithelial lesions. Regarding age, (30.61\%) of women was under 30 years old. The

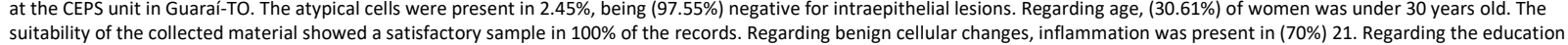
of women, complete high school predominated with (30.61\%). The predominant microbiological agent was Lactobacillus sp. com (80.82\%) of the results. Intraepithelial lesions had a low prevalence, compared to some literature. It can be seen that the health of patients at the CEPS unit in Guaraí, shows good suitability, since the numbers of agents that cause infection are reduced.
\end{abstract}

Keywords: Cytopathological examination; Woman; Cervix

Topic: Enfermagem em Saúde Pública

Reviewed anonymously in the process of blind peer
Received: 24/03/2021

Approved: 03/04/2021
Andréia Alves Teixeira

Faculdade Guaraí, Brasil

http://lattes.cnpq.br/3649435471229171

andreia.teixeira@hotmail.com

Dyéssika Rayane Alves dos Santos

Faculdade Guaraí, Brasil

http://lattes.cnpq.br/7519666484351261

dyessikarayane@hotmail.com

Glaucya Wanderley Santos Markus (iD)

Faculdade Guaraí, Brasil

http://lattes.cnpq.br/5568510365985231

http://orcid.org/0000-0001-8916-1086

glaucyamarkus@outlook.com
Adriana Keila Dias (D)

Faculdade Guaraí, Brasil

http://lattes.cnpq.br/2128882976477548

http://orcid.org/0000-0003-1291-5593

adrianakeiladias@hotmail.com

Giullia Bianca Ferraciolli do Couto (iD

Faculdade Guaraí, Brasil

http://lattes.cnpq.br/0109560699727614

http://orcid.org/0000-0002-9768-778X

giulliabianca@hotmail.com

Juliane Marcelino dos Santos (iD)

Faculdade Guaraí, Brasil

http://lattes.cnpq.br/8751832120632358

http://orcid.org/0000-0002-2059-1069

julianemarcelino@hotmail.com
Reobbe Aguiar Pereira (it)

Faculdade Guaraí, Brasil

http://lattes.cnpq.br/7447115724350334

http://orcid.org/0000-0003-2578-2611

enfreobbe@gmail.com

\section{Referencing this:}

TEIXEIRA, A. A.; SANTOS, D. R. A.; MARKUS, G. W. S.; DIAS, A. K.; COUTO, G. B. F; SANTOS, J. M.; PEREIRA, R. A.. Análise retrospectiva: o que denota os exames citopatológicos da unidade CEPS. Scire Salutis, v.11, n.2, p.93-101, 2021. DOI: http://doi.org/10.6008/CBPC22369600.2021.002.0010 


\section{INTRODUÇÃO}

O câncer do colo do útero (CCU) é a causa mais frequente de morte em mulheres em todo o mundo, no Brasil no ano de 2017, ocorreram 6.385 óbitos, e os índices de mortalidade de CCU foram de 6,17/100 mil. A estimativa mundial é de 570 mil novos casos. Em 2020, no Brasil, são esperados 16.590 novos casos e no estado do Tocantins apresenta um anunciado de 220 novos casos. Trata-se do terceiro tumor mais frequente na população feminina brasileira, e ocupa o quarto lugar entre os tipos de câncer (BRASIL, 2020; DIAS et al., 2019).

Dentre as técnicas de detecção do CCU, o processo de rastreamento é feito por meio do exame citopatológico, sendo esse reconhecido como uma estratégica barata, segura e eficaz. O principal objetivo é identificar precocemente e tratar as lesões em tempo oportuno antes da evolução para uma doença invasiva. Nesse sentido com $80 \%$ de cobertura da população-alva é possível obter redução dos índices de mulheres com lesões, e possivelmente redução de mortalidade por essa patologia (CORRÊA et al., 2017; GURGEL et al., 2019).

Segundo o Ministério da Saúde o rastreamento é indicado para mulheres na faixa etária de 25 a 64 anos, que já mantém atividade sexual ativa, a realização deve ser anual ou a cada três anos, após dois exames consecutivos com resultados negativos realizados anualmente. $\mathrm{O}$ exame preventivo é rápido e indolor, embora haja avanços tecnológicos para detecção do CCU o número ainda é alto de mulheres que não procuram a unidade de saúde para realização do exame (BRASIL, 2019; TAVARES et al., 2018).

Existem alguns fatores de risco que podem contribuir para o desenvolvimento do CCU como: os aspectos socioeconômicos, início precoce da atividade sexual, múltiplos parceiros sexuais, tabagismo e uso prolongado de pílulas anticoncepcionais. Outro importante fator de desenvolvimento dessa patologia é a infecção pelo papilomavírus humano (HPV), que está associada à maior parte dos casos de lesão precursora do câncer do colo do útero (SOUSA et al., 2017; SOUZA et al., 2015).

A Política de Atenção à Saúde da Mulher tem como método fortalecer as práticas de prevenção de CCU com ações educativas em saúde e ressaltando a importância do rastreamento. Com isso, o empenho do enfermeiro no combate da doença e essencial com medidas primaria e segundaria da população feminina, visando ações com objetivos de controle do CCU e na atuação de prevenção, detecção precoce, diagnóstico e tratamento (MELO et al., 2017).

No desenvolvimento da prática assistencial deve-se estimular a população feminina educação em saúde de modo integral, incentivar o autocuidado, por meio da realização do exame citopatológico, uso de anticoncepção, doenças sexualmente transmissíveis, sinais e sintomas. Essas medidas podem ser necessárias no processo de autoimagem e na sexualidade, e podem favorecer mudanças de comportamentos e de atitudes das mulheres. (COSTA et al., 2017; MELO et al., 2017; MONTEIRO et al., 2019).

A Clínica de Educação Para Saúde CEPS, criada por iniciativa do Instituto Educacional Santa Catarina - Faculdade de Guaraí (IESC/FAG), fundada em 2012, propõe atender a população que necessita de assistência à saúde. Na clínica o programa saúde da mulher visa assistência qualificada em cuidados, é as 
consultas de enfermagem são de grande importância com a finalidade de assegurar a mulher na linha de prevenção do CCU (FREIRE et al., 2016).

Esse artigo tem como objetivo o interesse de apresentar a prevalência de alterações dos resultados de exames citopatológicos das pacientes que realizaram consulta de enfermagem na clínica CEPS no municipal de Guaraí. Além disso, os objetivos secundários são apresentar o perfil etário das pacientes, grau de instrução e os principais achados referentes às alterações celulares, microbiológicas, e satisfação do material.

\section{METODOLOGIA}

Este estudo propôs a realização de uma pesquisa de campo de metodologia quantitativa e abordagem descritiva, os dados foram coletados pelas pesquisadoras, baseados nos achados de resultados de exames citopatológicos, a partir de análise de fichas ambulatoriais de pacientes assistidas na Clínica de Educação Para Saúde (CEPS), do Instituto Educacional Santa Catarina IESC/FAG, campos III, no município de Guaraí-TO.

A coleta dos dados ocorreu dia 09/09/2020 e 10/09/2020 no horário de funcionalidade da unidade CEPS, na análise retrospectiva foram obtidos 250 resultados de exames citopatológicos realizados entre o período de Março de 2018 a agosto de 2020. Nos critérios de inclusão utilizaram-se as variáveis referentes aos resultados de exames que apresentaram diagnóstico laboratorial descrito de forma clara e completa, idade entre 25 a 64 anos, grau de instrução, adequabilidade do material coletado, apitélios apresentados nas amostras, alterações celulares e microbiológicas.

Da totalidade de resultados, exclui-se 05 registros de exames por não constarem informações suficientes para os objetivos da pesquisa, apresentarem informações pessoais incompletas, resultados não encontrados na ficha ambulatorial, fichas de pacientes menores de idade.

Essa pesquisa não teve necessidade do termo de consentimento livre e esclarecido, pois as análises foram realizadas a partir de contabilização de resultados de exames citológicos, verificação de fichas ambulatoriais, livros de registros. Esse artigo cumpriu com toda obrigatoriedade de normas do comitê de ética. A pesquisa foi realizada com autorização de uso de dados pelo diretor do Instituto Educacional Santa Catarina do IESC/FAG, levando-se em consideração respeito aos princípios éticos constituídos pela Resolução COFEN no 564/2017 do Conselho Nacional de Saúde, garante-se confidencialidade dos dados, segurança dos prontuários; não divulgar informação e resultados com identificação de usuárias. A pesquisa obteve a aprovação do Comitê de Ética em Pesquisa do Instituto Federal do Tocantins - IFTO, campus de Palmas-TO, emitido sob o Parecer no 4.554.050.

\section{RESULTADOS E DISCUSSÃO}

Foram avaliados 245 laudos de exame citopatológico através de levantamentos dos dados nas fichas ambulatoriais das pacientes da unidade CEPS em Guaraí- TO, no ano de 2018 a 2020. Os resultados encontrados foram sistematizados e estruturados em tabelas e analisados diante a literatura. A Tabela 01 
refere-se à classificação percentual da faixa etária e escolaridade.

Tabela 01: Caracterização sócio demográfica das pacientes da unidade CEPS.

\begin{tabular}{ll}
\hline VARIÁVEL & (\%) \\
\hline Idade (anos) & $30,61 \%$ \\
\hline Inferior a 30 anos & $16,73 \%$ \\
31 a 36 anos & $14,29 \%$ \\
37 a 42 anos & $13,88 \%$ \\
43 a 48 anos & $9,80 \%$ \\
49 a 54 anos & $14,69 \%$ \\
Acima de 55 anos & \\
\hline Escolaridade & $3,27 \%$ \\
\hline Fundamental & $13,47 \%$ \\
Fundamental Incompleto & $30,61 \%$ \\
Ensino médio Completo & $5,71 \%$ \\
Ensino médio Incompleto & $10,61 \%$ \\
Superior & $13,47 \%$ \\
Superior Incompleto & $22,86 \%$ \\
Não Identificados & \\
\hline
\end{tabular}

Identificou-se que, a média de prevalência de mulheres que compareceu na unidade CEPS foi a faixa etária inferior a de 30 anos (30,61\%) conforme demostrado no Tabela I. O estudo de (SILVA et al., 2014) também corroboram com essa pesquisa onde $(76,8 \%)$ era de mulheres entre 25 a 64 anos, sendo que a faixa etária de 25 a 29 anos foi a mais predominante.

Segundo o Ministério da Saúde (OMS) os índices de CCU em mulheres jovens com idade abaixo de 25 anos são menores do que em mulheres mais velhas, prevalecendo nessa idade as infecções por HPV e lesões de baixo grau, conseguindo ser acompanhada e tratada conforme indicações ambulatoriais. No entanto, a incidência de risco mostra que o aumento de câncer nas mulheres está entre as faixas etárias de 30 e 39 anos, atingindo o pico na quinta ou sexta décadas de vida (INCA, 2016).

Com relação ao grau de instrução a maioria das mulheres que realizaram os exames citopatológicos possuía grau de instrução médio completo com $(30,61 \%)$ dos resultados, ensino fundamental incompleto $(13,47 \%)$, fundamental completo $(3,27 \%)$, ensino médio incompleto $(5,71 \%)$, superior completo $(10,61 \%)$, superior incompleto $(13,47 \%)$, é excluindo os exames não identificados com $22,86 \%$.

A pesquisa parece em partes com o estudo de (LOPES et al., 2016) onde (40\%) das mulheres também apresentam ensino médio com maior prevalência. No mesmo estudo demostra que, no Brasil os índices de mortalidade por CCU estão associados ao baixo fator socioeconômico, as mulheres que inclui baixa escolaridade são mais vulneráveis, pois pode defrontar com algumas dificuldades ao acesso de serviço em saúde assim como economia, questões culturais, sociais, medo, vergonha ou desconhecimento de importantes sinais e sintomas do câncer uterino.

Segundo a análise de (AKINYEMIJU et al., 2016) os fatores socioeconômico e educacional de mulheres da Rússia, México, China e África do sul relacionado ao rastreamento do CCU têm mostrado que o grau de instrução é maior, e que isso apresenta boa possibilidade de participação delas nos programas de rastreamento do câncer uterino.

Na decorrência dos estudos, 245 exames citopatológicos analisados na unidade CEPS, foram encontradas $100 \%$ de adequabilidades satisfatórias. Os resultados do estudo de (MORAIS, et al., 2015) 
também apresentou (99\%) da presença de material satisfatório.

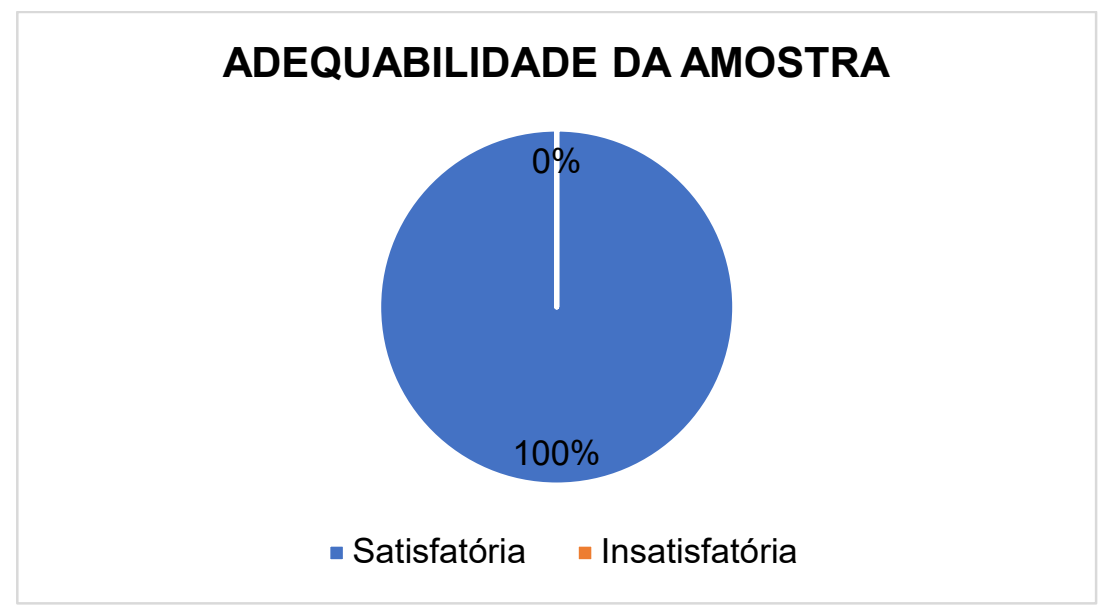

Gráfico 01: Adequação das amostras dos exames citopatológicos analisados no CEPS, Guaraí-TO.

Ainda segundo (MORAIS et al., 2015) a tendência do estudo que avalia a adequabilidade das amostras dos exames realizados do colo do útero identifica -se amostras satisfatórias aquelas que contêm células em quantidade representativas, que são bem disseminadas, fixadas, coradas, e assim permitem sua observação e conclusões diagnósticas. Estão inclusas em uma boa coleta, amostras celulares dos epitélios do colo do útero, sendo elas, células escamosas, glandulares e metaplásicas.

No exame citopatológico uma amostra é considerada insatisfatória quando há algum problema relacionado ao material coletado tornando impossível analisar a lamina é emitir um resultado. Sendo assim, o exame necessita ser repetido, o que provoca aumento dos gastos do procedimento é também requer que a mulher retorne para uma nova coleta. Então, deve-se considerar que a adequabilidade é condição do material está ligado à forma de coleta desse exame (RIBEIRO et al., 2018).

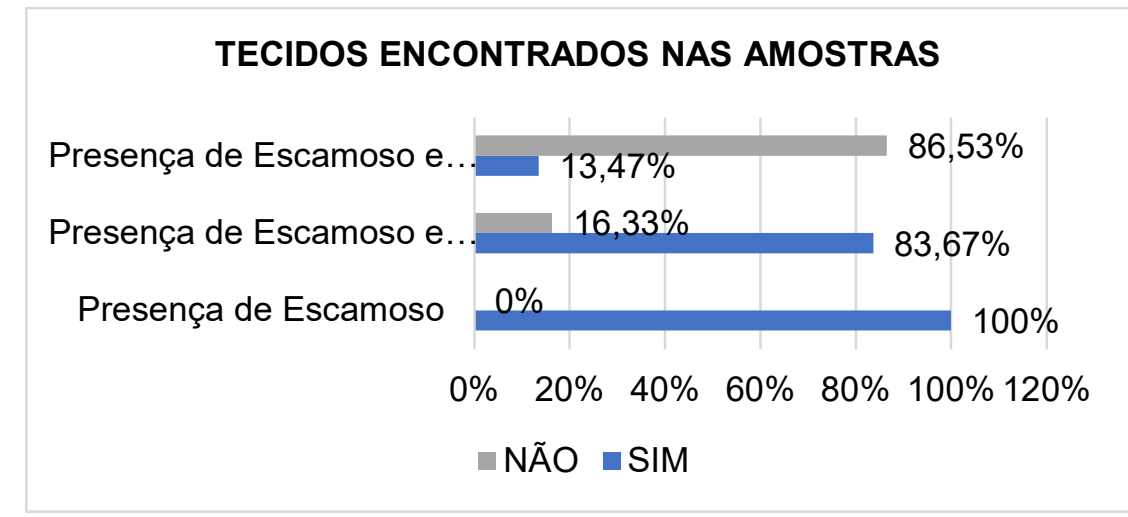

Gráfico 02: Frequência dos tecidos encontrados nas amostras dos exames citopatológicos analisados no CEPS, GuaraíTO.

No estudo realizado no CEPS, através de fichas ambulatoriais, consta que (100\%) 245 das amostras contêm epitélios possivelmente representados na amostra Escamoso, desses (83,67\%) 205 representaram escamoso e glandular, e apenas (13,47\%) 33 dos laudos apresentaram epitélios escamoso, glandular e metaplásico. Resultado semelhante encontrado no estudo de (GEREMIA et al., 2016), em que prevaleceu o epitélio escamoso com (100\%) 213, tecido glândulas (31,4\%) 67, e metaplásico consta em (9,8\%) 21, dos resultados. 
Para garantir a qualidade no laudo do exame citopatológico, espera-se que o mesmo apresente epitélio escamoso, glândulas e metaplásico, isso pode resultar em um diagnóstico falso negativo. Também vale mencionar que as células epiteliais descritas nos exames estão fortemente ligadas, incluindo métodos para a coleta de exames, determinação de materiais e preparação para teoria e prática profissionais, fatores que afetam diretamente na avaliação da amostra (SILVA et al., 2014).

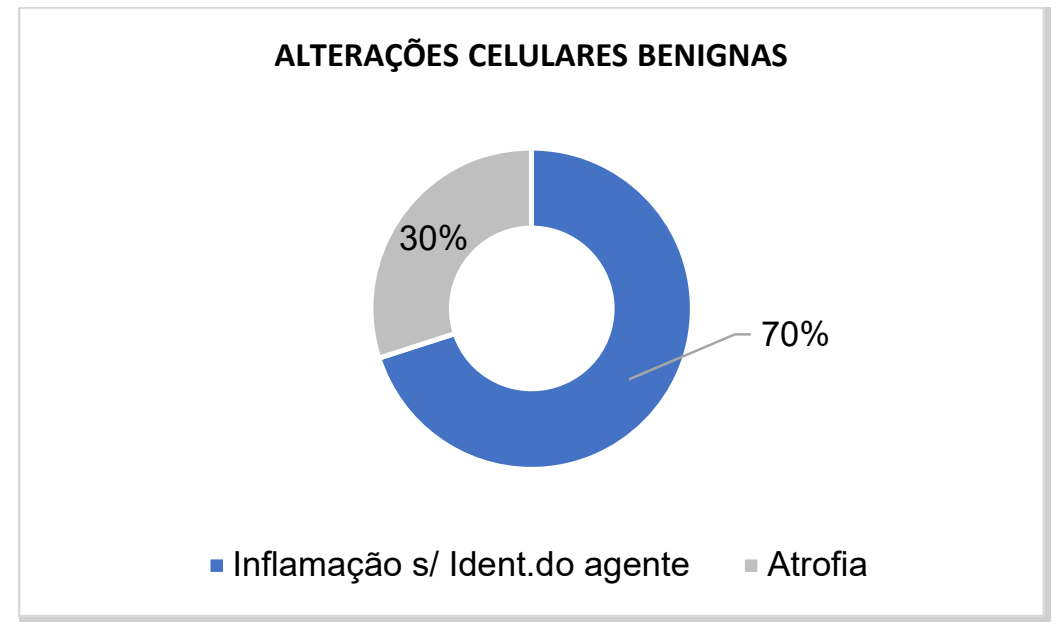

Gráfico 03: Alterações celulares benignas nas amostras dos exames citopatológicos analisados no CEPS, Guaraí-TO.

Nesse estudo, 70\% dos exames apresentam inflamações sem identificação do agente, e 30\% de atrofia. Esses estudos divergem com os resultados de (FREDRICH et al., 2019) nos quais as inflamações foram mais frequentes, com proporções de (47,5\%), hipotrofia 54 (2,3\%).

Segundo o (INCA, 2016). A presença de inflamação é considerada alta nos exames citopatológicos causando alterações nas células epiteliais do colo uterino, e canal vaginal, alguns fatores dessas inflamações ocorre pelo efeito de ações de agentes físicos (mecânico, térmico, radiativo) e químicos (medicamentos, cáusticos, quimioterápico, acidez).

Segundo (SILVA et al., 2014) o processo de inflamação pode afetar a qualidade da amostra, sendo melhor realizar o tratamento é após três meses a nova coleta citológica pode ser realizada. Vale destacar que se a segunda citologia da paciente apresentar positivo para inflamação é necessário encaminhar a mesma para realização de colposcopia.

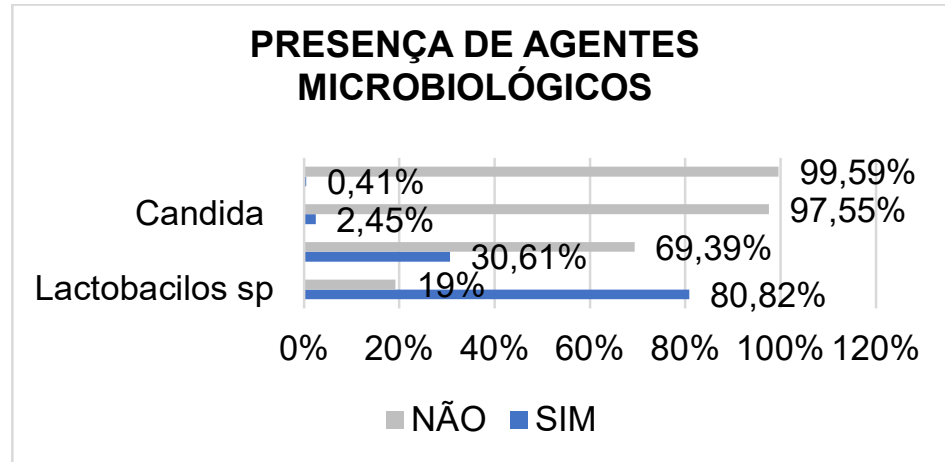

Gráfico 04: Avaliação da presença de agentes microbiológicos nas amostras dos exames citopatológicos analisados no CEPS, Guaraí-TO.

Referente à microbiologia Lactobacillus sp. foi representado com (80,82\%) 198 dos resultados, 
seguido por cocos e outros Bacilos (30,61\%), Candida sp. (2,45\%) 6, e Gardnerella vaginalis $(0,41 \%) 1$. Resultado que assemelha a este estudo é de (FERREIRA et al., 2015) onde Lactobacillus (39,33\%) também apresentou em maior número, Cocos e outros Bacilos (22,85\%).

Segundo (GEREMIA et al., 2016). Lactobacilos, Cocos, outros Bacilos, são achados normais que fazem parte da flora vaginal, e essas características não necessitam de tratamento. Os microrganismos infecciosos que apresentaram nesse estudo foram Candida sp., e Gardnerella vaginalis, ambas são classificadas como vulvovaginites.

A vaginose é caracterizada por uma bactéria que se prolifera causando desequilíbrio na flora normal da vaginal, sendo elas as anaeróbias - Gardnerella vaginalis, Bacteroides sp., Mobiluncus sp., o aumento dessas bactérias estão ligados na falta ou diminuição dos lactobacilos. Já a candidíase é uma infecção da vulva e vagina caudada por fungos que reside na mucosa vaginal e que cresce quando o meio é favorável para o seu desenvolvimento (FERREIRA et al., 2015).

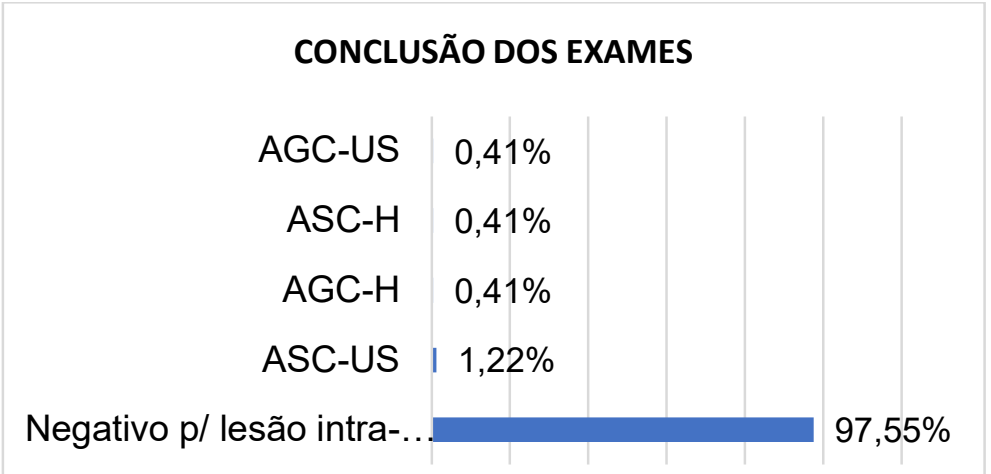

0\% 20\% 40\% 60\% 80\%100\% $20 \%$

Gráfico 05: Prevalência de resultados dos exames citopatológicos analisados no CEPS, Guaraí-TO.

*ASC-US: atipia escamosa de significado indeterminado, que podem ser não a neoplásicas; *ASC-H: atipia escamosa de significado indeterminado, não descarta lesão de alto grau; *AGC-H: atipia glandular de significado indeterminado, não afastando lesão de alto grau não podem ser afastados *AGC-US: atipia glandular de significado indeterminado, possivelmente não neoplásica.

Os resultados analisados nas fichas ambulatoriais realizados no CEPS demonstram que do total de 245 mulheres, (97,55\%) apresentaram resultado negativo para lesão intraepitelial, ASCU-S apenas 2 (1,22\%), AGC-H 1 (0,41\%), ASC-H 1 (0,41\%), AGC-US 1 (0,41\%). No entanto, o estudo realizado por (DALMOLIN et al., 2016), demonstra divergência ASCU-S com 696 (72,86\%) com maior prevalecendo dos resultados.

No estudo de (BATISTA et al., 2012) diz que os diagnósticos de ASC-H são mais comuns em mulheres mais velhas, é ASC-US exibe maior frequência em mulheres jovens. Embora, essas lesões de baixa gravidade apresentam em menores índices na população jovial, os riscos de torna-se câncer e grande, caso não seja monitorados e previamente tratados em tempo oportuno.

Segundo (ROCHA et al., 2016) as atipias escamosas de significado indeterminado mostra com frequência a variedade de atipia descrita nos laudos citopatológicos, recomenda-se que fique abaixo de 5\% 
do total de exames realizados, em um só laboratório. No levantamento da pesquisa, a frequência apresentou abaixo da média. No estudo de (DIAS et al., 2019) mostra que os números elevados de ASC-US podem estar relacionados nas dificuldades de diagnóstico recorrente á problemas na coleta citológica, análise das lâminas e barreiras nos serviços públicos de saúde.

\section{CONCLUSÕES}

Nesse estudo foi possível observar que a prevalência de mulheres que procuraram a unidade CEPS teve faixa etária inferior a 30 anos de idade, estando dentro do padrão preconizada pelo MS para a realização do exame citopatológico.

Percebe-se que, a saúde das pacientes da unidade CEPS de Guaraí exibem boa adequabilidade, visto que os números de agentes que causam infecção estão reduzidos são as lesões intraepiteliais apresentaram baixa prevalência em comparado com algumas literaturas assim, o papel do enfermeiro é fundamental para conscientizar a população feminina dos benefícios da prevenção e incentivar a realização do exame citopatológico, com vistas a reduzir a incidência do CCU.

Os laudos citopatológicos avaliados na clínica apresentaram características satisfatórias, evidenciando que as coletas e envios para o laboratório estavam sendo feitas de modo correto pelos profissionais e acadêmicos que realizam o exame, uma vez que a adequabilidade do material é importante para desfecho dos diagnósticos. Não foi encontrada amostra insatisfatória, porém, alguns laudos exibiram ausência de células metaplásicas sendo necessário avaliar a técnica de coleta citológica, a fim de obter melhores resultados nas amostras e consequentemente bons laudos dos exames.

Diante do exposto, a busca efetiva em implementações, visa a qualidade de assistência à saúde da mulher, além de contribuir para novos conhecimentos e aguçar novas pesquisas sobre a temática. Outrossim, essa pesquisa visa contribuir para melhoria nos empenhos dos profissionais e acadêmicos quanto ao manejo das coletas citopatológicas, e garantir a eficiência do exame preventivo das mulheres submetidas ao teste.

\section{REFERÊNCIAS}

AKINYEMIJU, T.. Life-course socioeconomic status and breast and cervical cancer screening: analysis of the who's Study on Global Ageing and Adult Health (SAGE). BMJ Open, v.6, n.11, p.1-10, 2016

BATISTA, M. L. S.. Resultados citopatológicos de mulheres que realizaram exame do colo do útero em um laboratório escola da Universidade Federal de Goiás. J Health Sci Inst, v.30, n.3, p.201-205, 2012.

BRASIL. Diretrizes brasileiras para o rastreamento do câncer do colo do útero. Rio de Janeiro: INCA, 2016.

BRASIL. Estimativa 2020: incidência de câncer no Brasil. Rio de Janeiro: INCA, 2019.

BRASIL. Diretrizes brasileiras para o rastreamento do câncer do colo do útero. Rio de Janeiro: INCA, 2019.
CORRÊA, C. S. L.. Rastreamento do câncer do colo do útero em Minas Gerais: avaliação a partir de dados do Sistema de Informação do Câncer do Colo do Útero (SISCOLO). Revista Caderno de Saúde Coletiva, v.25, n.3, p.315-23, 2017.

COSTA, F. K. M.. Os desafios do enfermeiro perante a prevenção do câncer do colo do útero. Revista Gestão \& Saúde, p.55-62, 2017.

DALMOLIN, S. P.; DEXHEIMER, G. M.; DELVING, L. K. O. B. Mulheres com exames citopatológicos alterados: avaliação do seguimento de acordo com as condutas preconizadas pelo ministério da saúde. Revista Brasileira de Análises Clínicas, v.48, p.235-239, 2016.

DIAS, C. F.. Perfil de exames citopatológicos coletados em estratégia de saúde da família. Rev. Fun. Care, v.11, n.1, p.192-198, 2019. 
FERREIRA, J. E. L.; ALVES, M. C.; MARTINS, M. C. V.; SANTA, R. M. P. R.; GONÇALVES, M. C.. Perfil da população atendida em um consultório de atendimento integral á saúde da mulher. Caderno de Graduação - Ciências Biológicas e da Saúde - UNIT, v.3, n.1, p.127-140, 2015.

FREDRICH, É. K.; RENNER, J. D. P.. Alterações citopatológicas em exames de Papanicolau na cidade de Santa Cruz do Sul, Rio Grande do Sul, Brasil. J. Bras. Patol. Med. Lab., v.55, n.3, p.246-257, 2019

FREIRE, R. M. A.. Um olhar sobre a promoção da saúde e a prevenção de complicações: diferenças de contextos. Rev. Latino-Am. Enfermagem, v.24, 2016

GEREMIA, D. A.; DERNER, T.; ROSA, M.; ANTUNES, T. R.; GASPARIM, V. A.. Avaliação da adequabilidade da coleta do exame citopatológico na Estratégia Saúde da Família. Revista ACRED, v.6, n.11, p.99-108, 2016.

GURGEL, L. C.. Percepção de mulheres sobre o exame de prevenção de colo de útero Papanicolau: uma Revisão Integrativa da Literatura. Ld. Rev.Mult. Psic, v.13, n.46, p.434-445, 2019.

LOPES, N. G.. Avaliação da eficácia do exame de rastreamento de lesões hpv em mulheres. Rev Enferm UFPE, v.10, n.4, p.1292-1298, 2016.

MELO, F. B. B.. Actions of nurses in early detection of breast cancer. Rev. Bras. Enferm, v.70, n.6, p.1119-1128, 2017.

MONTEIRO, N. J.. Avaliação do serviço de coleta para exame colpocitológico pela escala SERVQUAL. Rev. Bras. Enferm., Brasília, v.72, n.1, p.118-124, 2019.
MORAIS, M. N.; JERÔNIMO, C. G. F.. Análise dos resultados de exames citopatológicos do colo uterino. Revista de Enfermagem UFPE, v.9, n.3, p.7510-7515, 2015.

ROCHA, S. M. M.; BAHIA, M. O.; ROCHA, C. A. M.. Perfil dos exames citopatológicos do colo do útero realizados na Casa da Mulher, Estado do Pará, Brasil. Rev Pan-Amaz Saude, v.7, n.3, p.51-55, 2016

RIBEIRO, W. A.; ANDRADE, M; FASSARELLA, B. P. A.; SANTANA, P. P. C.; COUTINHO, V. V. A.; SPERENDIO, L.; PINHEIRO, D. S.. Análise das amostras insatisfatórias do exame citopatológico do colo do útero nas regiões brasileiras: um estudo retrospectivo. Revista Pró-UniverSUS. v.9, n.2, p.53-59, 2018.

SILVA, D. S. M.. Rastreamento do câncer do colo do útero no Estado do Maranhão, Brasil. Rev. Ciência \& Saúde Coletiva, v.19, n.4, p.1163-1170, 2014.

SOUSA, A. C. O.. Análise das alterações citopatológicas registradas no sistema de informação do câncer de colo do útero em Teresina. R. Interd., Teresina, v.10, n.4, p.21-30, 2017.

SOUZA, A. F.; COSTA, L. H. R.. Conhecimento de Mulheres sobre HPV e Câncer do Colo do Útero após Consulta de Enfermagem. Revista Brasileira de Cancerologia, v.61, n.4, p.343-350, 2015.

TAVARES, D. I.; SCHEID, G. S.; BRAZ, M. M.. Prevalência do exame citopatológico cérvico-vaginal e microflora em idosas de Santa Maria, Rio Grande do Sul. Revista Saúde, v.44, n.1, p.1-8, 2018.

A CBPC - Companhia Brasileira de Produção Científica (CNPJ: 11.221.422/0001-03) detém os direitos materiais desta publicação. Os direitos referem-se à publicação do trabalho em qualquer parte do mundo, incluindo os direitos às renovações, expansões e disseminações da contribuição, bem como outros direitos subsidiários. Todos os trabalhos publicados eletronicamente poderão posteriormente ser publicados em coletâneas impressas sob coordenação da Sustenere Publishing, da Companhia Brasileira de Produção Científica e seus parceiros autorizados. Os (as) autores (as) preservam os direitos autorais, mas não têm permissão para a publicação da contribuição em outro meio, impresso ou digital, em português ou em tradução. 